\title{
Choquequirao en el siglo XVI: etnohistoria e implicaciones arqueológicas
}

Choquequirao au XVI siècle : ethnohistoire et implications archéologiques

Choquequirao in the $16^{\text {th }}$ century: ethnohistory and archaeological implications

\section{Erwan Duffait}

\section{(2) OpenEdition}

\section{Journals}

Edición electrónica

URL: http://journals.openedition.org/bifea/5467

DOI: $10.4000 /$ bifea. 5467

ISSN: 2076-5827

Editor

Institut Français d'Études Andines

Edición impresa

Fecha de publicación: 1 agosto 2005

Paginación: 185-196

ISSN: 0303-7495

Referencia electrónica

Erwan Duffait, « Choquequirao en el siglo XVI: etnohistoria e implicaciones arqueológicas », Bulletin de I'Institut français d'études andines [En línea], 34 (2) | 2005, Publicado el 08 agosto 2005, consultado el 02 diciembre 2020. URL : http://journals.openedition.org/bifea/5467 ; DOI : https://doi.org/10.4000/ bifea.5467

\section{(c) $(1)$}

Les contenus du Bulletin de l'Institut français d'études andines sont mis à disposition selon les termes de la licence Creative Commons Attribution - Pas d'Utilisation Commerciale - Pas de Modification 4.0 International. 


\title{
Choquequirao en el siglo XVI: etnohistoria e implicaciones arqueológicas*
}

\author{
Erwan Duffait**
}

\section{Resumen}

Este trabajo, a través del análisis de varias fuentes manuscritas, reconstituye la historia colonial del sitio arqueológico de Choquequirao. En primera instancia encomienda de Hernando Pizarro, este sitio fue reducido a fines del siglo XVI en el pueblo de Cachora, junto con los pueblos de Sayhuite y Urco. Este artículo demuestra también que los españoles concedieron el cargo de cacique de estos pueblos a las mismas familias durante más de 80 años. Estos nuevos conocimientos sobre la historia de Choquequirao llevan a plantear nuevas preguntas a la arqueología.

Palabras clave - Perú, época colonial, cultura Inca, encomienda, reducción, arqueología

\section{Choquequirao au XVle siècle : ethnohistoire et implications archéologiques}

\section{Résumé}

À travers l'étude de divers documents d'archives, ce travail reconstitue l'histoire coloniale du site archéologique de Choquequirao. Ce site, qui faisait tout d'abord partie d'une encomienda octroyée à Hernando Pizarro, devient partie intégrale de la réduction de Cachora, à la fin du XVIe siècle, avec les anciens villages de Sayhuite et Urco. Cet article démontre aussi que durant plus de 80 ans, les

Quisieramos agradecer a Thérèse Bouysse-Cassagne por sus valiosos consejos así como a Jean-François Bouchard y Patrice Lecoq por sus sugerencias. También deseamos dar las gracias a Renata García Moreno por la revisión del texto en español.

** Doctorant à I'Université Paris I, Panthéon Sorbonne - UMR 8096 du CNRS «Archéologie des Amériques». Pour communication, écrire: Centre d'Archéologie Précolombienne, Université Paris I, 3, rue Michelet 75006 Paris. Correo electrónico: erwanduffait@yahoo.fr 
Espagnols ont concédé aux mêmes familles les charges de cacique de ces villages. Ces nouvelles données concernant I'histoire de Choquequirao amènent à se poser de nouvelles questions dans le domaine de l'archéologie.

Mots clés - Pérou, époque coloniale, culture Inca, encomienda, réduction, archéologie

\title{
Choquequirao in the $16^{\text {th }}$ century: ethnohistory and archaeological implications
}

\begin{abstract}
Through the study of various archive documents, this work reconstructs the colonial history of the archaeological site of Choquequirao. This site, which first formed part of a Hernando Pizarro's holding, was reduced together with the old villages of Sayhuite and Urcp to the locality of Cachora at the end of the 16th century. This article also shows that during more than 80 years, the Spaniards conceded the office of cacique of these villages to the same families. These new data concerning the history of Choquequirao bring to raise new questions for archaeology.
\end{abstract}

Key words - Peru, colonial time, Inca culture, encomienda, reduction, archaeology

En el siglo XIX, el sitio arqueológico de Choquequirao tenía fama de ser el último baluarte de los incas refugiados en la cordillera de Vilcabamba entre 1537 y 1572 y, hoy día, estos relatos permanecen vigentes. Sin embargo, gracias a la «Instrucción» de Titu Cusi Yupanqui (1916[1570]) y a varias cartas intercambiadas entre españoles e incas de Vilcabamba, llegamos a conocer los sitios donde se habían establecido Manco Inca y sus hijos. Estos sitios eran Vitcos y Vilcabamba, ubicados en la ladera norte de la cordillera de Vilcabamba, muy alejados de las tierras controladas por los españoles (fig. 1). Además, a la luz de otros documentos de los siglos XVI y XVII, se puede asegurar que Choquequirao era conocido y controlado por los españoles.

Hace poco, Rafael Varón Gabai $(1996 ; 1998)$ y Catherine Julien $(1998 ; 2001)$ publicaron documentos muy valiosos sobre las encomiendas que recibió Hernando Pizarro en 1539. En la región del Cusco, Hernando Pizarro tenía encomiendas importantes en los valles del Urubamba y del Apurímac. En el valle del Urubamba, su encomienda abarcaba los pueblos de Tambo (Ollantaytambo) y Piccho (o Pisco), o sea el sitio actual de Machu Picchu (Glave \& Remy, 1983: 2; Varón Gabai, 1996: 320; Julien, 2001: 249-250; Rowe, 1987: 15). Al sur, en el valle del Apurímac, Hernando Pizarro tenía varias tierras con los pueblos de Sayhuite, Choquequirao y Urco (Vaca de Castro 1908 [1543]: 443; Varón Gabai, 1996: 320-321; Julien, 2001: 249-250). Sin embargo, a fin de identificar con seguridad el sitio de Choquequirao, así como Sayhuite y Urco en los manuscritos publicados por Julien y Varón Gabai, se necesita confrontar y analizar dichos manuscritos con otras fuentes y documentos que se encuentran en varios archivos peruanos. 
En abril del año 1539, Francisco Pizarro otorgó a su hermano Hernando varias tierras en el valle del Apurímac entre las cuales se encontraban: «el pueblo de Urco de ques caçique Curima» [o Curiana, o Carima, según las copias del manuscrito] (Julien, 2001: 249, 251, 253; Varón Gabai, 1996: 320, 336). Este pueblo de Urco es el mismo que fue mencionado por Vaca de Castro (1908 [1543]: 443), perteneciendo a Hernando Pizarro y ubicado cerca del tampu de Curahuasi. Sin embargo, no llegamos a ubicar este pueblo. Actualmente, en la zona de Curahuasi, dos sitios tienen un topónimo parecido a Urco (o Urcon) y podrían corresponder a este pueblo antiguo. El primero es el cerro Orconcito (Instituto Geográfico Militar, 1946), ubicado frente a Choquequirao en el margen izquierdo del Apurímac. En la cumbre del cerro se encuentra un sitio arqueológico llamado Incahuasi o Incaraqay (fig. 1).

Este sitio fue mencionado por primera vez por Charles Wiener en el siglo XIX. En este lugar, el explorador francés notó la presencia «de tres palacios alineados de Norte a Sur», así como algunos andenes y otras «construcciones antiguas [...] [desplomadas] por completo» (Wiener, 1993 [1880]: 308). Las tres estructuras alineadas siguen de pie y recientemente, Copesco, el organismo cuzqueño encargado de la puesta en valor de Choquequirao desde 1993, hizo el levantamiento topógrafico de estos tres recintos. Se trata de edificios rectangulares de arquitectura típicamente Inca, con dos vanos de acceso cada uno, orientados hacia el oeste.

La tasa del repartimiento de Urcon, en 1557, nos informa que los indios entregaban ají en tributo (Varón Gabai, 1996: 336; Julien, 2001: 268). Esta planta necesita condiciones climáticas calientes y secas a fin de crecer. En la zona de Curahuasi, estas condiciones se encuentran únicamente en el valle del Apurímac y la parte baja de las quebradas que desembocan en dicho valle. El cerro Orconcito, por su localización, permite el cultivo del ají y el sitio de Incahuasi podría corresponder al pueblo antiguo de Urco. Existe un segundo sitio que podría identificarse también a Urco. A dos kilometros al sur de Sayhuite está ubicado el cerro Orcoñi en la cumbre del cual se encuentra el sitio arqueológico epónimo, constituido por una plaza rodeada de recintos y una plataforma de tipo ushnu (Kendall, 1980: 2). Sin embargo, la altura (3 200 m.s.n.m) y el clima húmedo y frío de la zona (Fornee, 1965 [1586]: 26) no permiten el cultivo del ají.

El pueblo de «Xuybita [con su] caçique Ynesnache» (Julien, 2001: 250; Varón Gabai, 1996: 320) formaba también parte de la encomienda de Hernando Pizarro. Xuybita es una deformación de Saiuita o Saiuite (Fornee, 1965 [1586]: 27) y se identifica al sitio arqueológico actual de Sayhuite, ubicado al oeste de Curahuasi y al sur de Cachora. Por fin, fue encomendado a Hernando Pizarro el pueblo que «se llama Chuquicarando [o Chuquierrando] en donde tiene su casa Atapoma con todos los yndios e prinçipales a el subjetos» (Julien, 2001: 250, 252, 254; Varón Gabai, 1996: 321). Lo más probable, es que Chuquicarando sea una deformación de Choquequirao, lo que nos indicaría que Choquequirao era el nombre original del sitio. Además, cabe señalar que el nombre del pueblo perteneciente a Hernando Pizarro mencionado por Vaca de Castro (1908 [1543]: 443) se llama Chuquitambo. Sin embargo, se trata muy posiblemente del mismo sitio, o sea del pueblo de Choquequirao.

\section{LA REDUCCIÓN EN EL PUEBLO DE CACHORA}

En la Biblioteca Nacional del Perú, se encuentra un manuscrito que permite confirmar estas primeras observaciones. El documento trata de una repartición de tierras ubicadas frente a Choquequirao, en la ribera izquierda del Apurímac, en la zona de Huanipaca y Cachora. Este documento, del año 1618, nos indica que los caciques del pueblo de Cachora eran: «don Salvador Achic gouernador de los yndios del $\mathrm{pu}^{\circ}$ de Cachora y don Fran(co) Atao Uuco, don Martin Ataopoma, don Fran(co) Layme [y] don Juan Quisoruco» (BNP, B 753, año 1618, fol. 28v). El cacique con el cargo más alto de gobernador, se llamaba Achic. El segundo cacique en la jerarquía era «don Martin Ataupoma segunda persona deste pueblo» (BNP, B 753, año 1618, fol. 32v). 


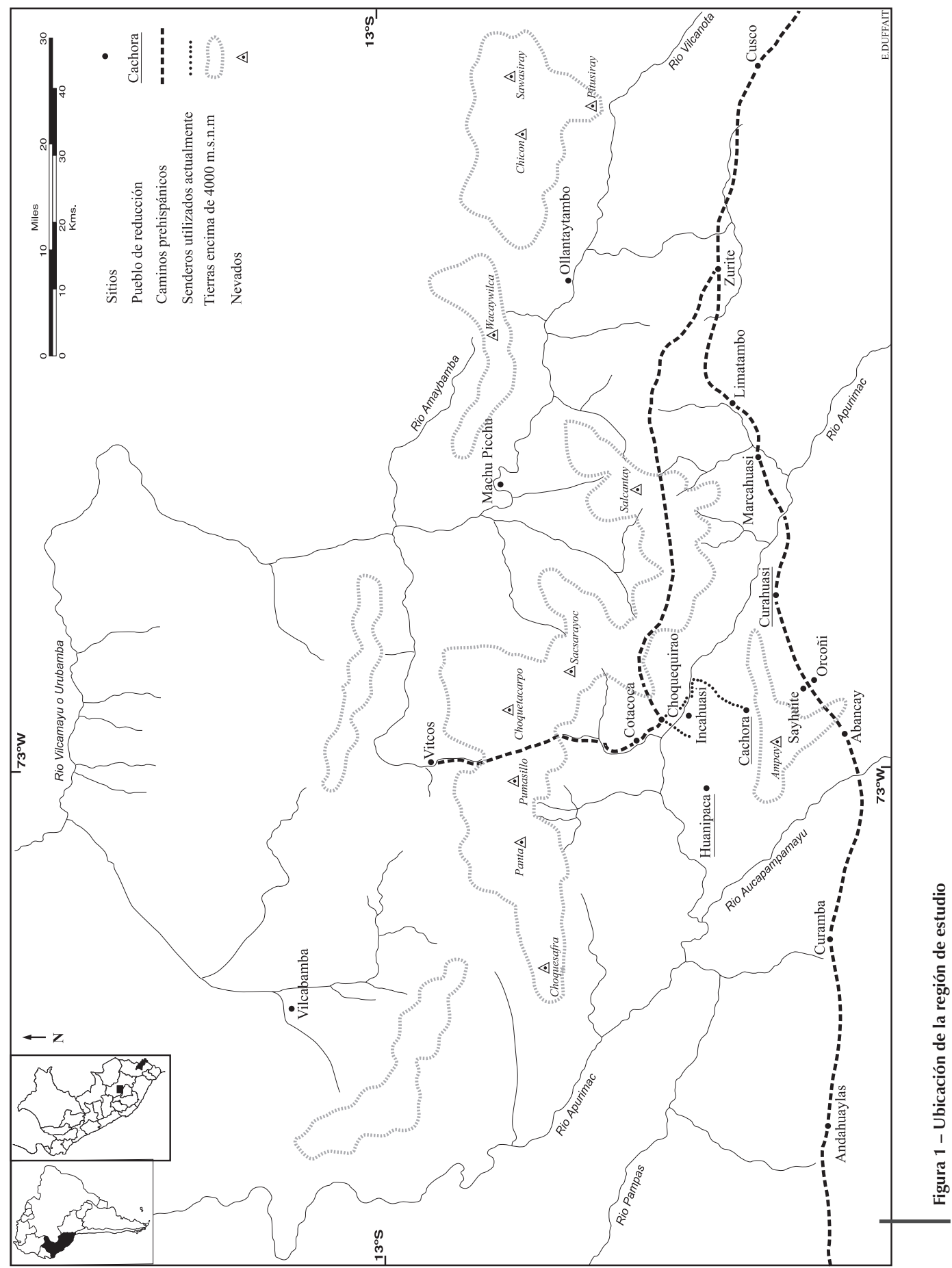


Los nombres Achic (o Ache) y Ataopoma, en 1618, son los mismos que encontramos en 1539 como apellidos de los caciques de Sayhuite y Choquequirao: Ynesnache y Atapoma, si suponemos que Ynesnache es una deformación de Ache. Se trataría, sin duda, de las mismas familias. Estos datos parecerían indicar que Cachora fue el pueblo de reducción de estos dos pueblos. En efecto, en su artículo sobre la hacienda Punchaopuquio, «tierras del sol» en la época Inca y ubicadas entre Sayhuite y Curahuasi, Donato Amado Gonzáles (1999: 40) indica que «[en el] pueblo de San Pedro de Cañete de Cachora está reducido el pueblo viejo de Saybita», lo que parece confirmar esta idea. Además, hoy día, según las creencias locales, los antiguos pobladores de Cachora vinieron de Sayhuite y Choquequirao (Miranda Valenzuela, 2002: 473).

Por otro lado, en el tercer pueblo llamado Urco (o Urcon), se nota la presencia de dos caciques en 1557: «don Hernando Ata Ulluco e don Françisco Curiata» (Varón Gabai, 1996: 336; Julien, 2001: 268). En la lista de caciques de Cachora, en 1618, se hace referencia a don Françisco Atao Uuco. Este personaje es, sin duda, un familiar de Hernando Ata Ulluco. Así, a la luz de estos datos, se puede plantear la hipótesis de que Cachora sería el pueblo de reducción de tres antiguos pueblos: Sayhuite, Choquequirao y Urco (cuadro 1). De hecho, al fin del siglo XVIII, el pueblo de Cachora tenía por nombre: «el pueblo de Cachora Orconsaibite» (AGN, Derecho Indigena, L. 25, C. 451, año 1791, fol. 7r).

Cuadro 1 - Los pueblos de Sayhuite, Choquequirao y Urco reducidos en Cachora

\begin{tabular}{|c|c|c|}
\hline FECHA & PUEBLO & CACIQUE \\
\hline 1539 & Sayhuite [Xuybita] & Ynesnache \\
\hline & $\begin{array}{c}\text { Choquequirao } \\
\text { [Chuquicarando] }\end{array}$ & Atapoma \\
\hline 1557 & Urco [o Urcon] & Curiana \\
\hline 1618 & Urco [o Urcon] & Curiata y Ataulluco \\
\hline 1637 & Cachora & $\begin{array}{c}\text { Ache, Ataopoma, Ataouco, } \\
\text { Layme y Quisoruco }\end{array}$ \\
\hline
\end{tabular}

Fuentes - Varón Gabai, 1996: 320-321; Julien, 2001: 249-250,268; BNP, B 753, año 1618; ADC, Cabildo, L. 3, C.25, año 1637

Aunque desconocemos de momento la fecha de fundación exacta del pueblo de reducción de Cachora, se podría proponer una fecha aproximada a la luz de los datos siguientes. En 1586, el corregidor Niculoso de Fornee (1965 [1586]: 27) menciona todavía la existencia del pueblo de «San Pedro de Saiuita». Eso parece indicar que el pueblo de reducción de Cachora fue fundado después de esta fecha. El nombre mismo del pueblo podría darnos un indicio en cuanto a su fecha de creación. Su nombre completo es San Pedro de Cañete de Cachora (Amado Gonzáles, 1999: 40). Cañete es, tal vez, una referencia a García Hurtado de Mendoza, marqués de Cañete y virrey del Perú entre 1590 y 1596. Así, podríamos plantear la hípotesis de que Cachora habría sido fundado en la época de dicho Virrey. La familia Ache, que tenía el cacicazgo en Sayhuite, obtuvo el cargo más alto de los caciques de Cachora. En 1637, esta familia ocupaba todavía este cargo de gobernador y cacique principal del pueblo de Cachora, en la persona de don Francisco Ache (ADC, Cabildo, L. 3, C. 25, año 1673, fol. 2r). En el momento de la reducción en el pueblo de Cachora, el cargo de segunda persona fue atribuido a los caciques de Choquequirao que ocupaban un rango menor en la jerarquía. 
Otro documento que trata de la zona de Huanipaca y Cachora parece indicar que estos dos pueblos fueron efectivamente creados al final de los años 1580, o al principio de los años 1590. Estos dos pueblos son los más importantes de la región, en esta parte del valle del Apurímac. Huanipaca y Cachora no aparecen en ningún documento anterior a 1586, por lo menos. Según un manuscrito ubicado en los Archivos Departamentales del Cusco, la primera referencia sobre Huanipaca data de 1587 (ADC, Colegio de Ciencias, L. 11, C. 15, años 1654-1825, fol. 17r). San Pedro de Cachora corresponde al pueblo de reducción de Sayhuite, Choquequirao y Urco, mientras que San Miguel de Huanipaca es la reducción de todos los pueblos antiguos, ubicados entre Huanipaca y la confluencia de los ríos Apurímac y Aucapampamayu.

Cabe señalar que desconocemos totalmente los nombres de los pueblos que fueron reducidos en Huanipaca. Sin embargo, mediante el documento del año 1618 que hemos utilizado anteriormente, tenemos confirmación de que este pueblo era efectivamente un pueblo de reducción: «los yndios de todos los ayllos reducidos en el $\mathrm{pu}^{\circ}$ de Guanipaca y Auancay de la encomienda de Gaspar de Sotelo» (BNP, B 753, año 1618, fol. 36r). El dicho Gaspar de Sotelo fue uno de los conquistadores de Vilcabamba en 1572, penetrando en la cordillera de Vilcabamba desde su encomienda, entre Curamba y Abancay (Murúa, 1962 [1611]: 249). Estos elementos nos indican que Cachora y Huanipaca habrían sido creados al fin de los años 1580 o principios de los años 1590. Podemos deducir, de todos estos datos, que Choquequirao fue abandonado muy posiblemente después de 1590, o sea, por lo menos más de 50 años después de la llegada de los españoles en Perú.

En la práctica, el proceso de las reducciones se manifestó de distintas formas. El reagrupamiento de varios pueblos en un pueblo de reducción no se tradujo sistemáticamente e inmediatamente por el abandono total de los antiguos pueblos. En realidad, sabemos que, muchas veces, los indios se quedaron en sus pueblos e iban al pueblo de reducción únicamente para asistir a la misa, vender sus productos al mercado y entregar el tributo (Matienzo, 1967: 48-56; Toledo, 1989: 242). En cuanto a Cachora, podemos sugerir dos posibilidades. Si la reducción en este pueblo hubiese sido un éxito inmediato, Choquequirao habría sido abandonado durante los años 1590. De lo contrario, los indios de Choquequirao (o una parte) habrían seguido viviendo en el sitio que fue abandonado más tarde. Si el segundo caso corresponde a la realidad histórica, no podemos proponer una fecha de abandono con base en los documentos conocidos. Siguiendo esta hipótesis, los habitantes de Choquequirao habrían podido quedarse en el sitio durante una parte del siglo XVII. Sea como fuere, el sitio estaba totalmente despoblado cuando Juan Arias Díaz Topete reconoció Choquequirao en 1710 (Huertas Vallejos, 1972: 204).

El elemento que destaca y que podemos observar a la luz de este análisis, se refiere a la continuidad en la atribución de los cargos de caciques. Desde 1539 (1557 en el caso de Urcon) hasta 1618, o sea un lapso de casi 80 años, son las mismas familias que tienen estos cargos: las familias Ache de Sayhuite (hasta 1637 por lo menos), Ataopoma de Choquequirao, y Atao Ulluco de Urcon. Se puede notar también la fecha tardía de la fundación de Cachora como de Huanipaca después del gran movimiento de las reducciones en la época de Francisco de Toledo (1569-1581).

\section{EL FRACASO DE LA PRIMERA REDUCCIÓN EN EL PUEBLO DE SALAMANCA}

Francisco de Toledo inició el gran movimiento de las reducciones durante su virreinato entre 1569 y 1581. En el valle del Apurímac, los dos repartimientos de Curahuasi, encomendados a Juan Gómez y Pedro de Cisneros, fueron reducidos en el pueblo actual de Santa Catalina de Curahuasi, mientras que los repartimientos de Urcon y Tayroma, encomendados respectivamente a Arias Maldonado (encomendero desde 1564) y Catalina Duarte, fueron reducidos en el pueblo de Salamanca (Miranda, 1975 [1583]: 183-185; Miranda, 1906 [1583]: 211; Puente Brunke, 1992: 352-353, 378-379). Así, el repartimiento de Urcon, constituido por varios pueblos (Urcon, 
Sayhuite y Choquequirao por lo menos), fue reducido primero en este pueblo de Salamanca conjuntamente con el pueblo pequeño de Tayroma. De hecho, 252 tributarios y 1802 personas vivían en el repartimiento de Urcon, mientras que solamente 25 tributarios y 163 personas vivían en el pueblo de Tayroma (Miranda, 1975 [1583]: 184-185). Aunque desconocemos la localización geográfica de Tayroma y Salamanca, esta reducción toledana fue un fracaso, ya que el repartimiento de Tayroma existía todavía en 1791 (AGN, Derecho Indigena, L. 25, C. 451, fol. 7 r) y el repartimiento de Urcon fue reducido una segunda vez en Cachora, probablemente durante el virreinato de García Hurtado de Mendoza, entre 1590 y 1596, como lo suponemos.

\section{LA ENCOMIENDA DE HERNANDO PIZARRO EN EL VALLE DEL APURIMAC: ¿PROPIEDAD PRIVADA DE LA DINASTÍA INCA?}

La encomienda otorgada a Hernando Pizarro en el valle del Apurímac en 1539 fue reclamada por el Inca Sayri Tupac, durante las negociaciones entre el dicho Inca y la Corona española. Sayri Tupac pedía todas las tierras ubicadas en el valle del Apurímac, río abajo del puente del Apurímac (entre Marcahuasi y Curahuasi) hasta la confluencia con el río Aucapampamayu, sobre una banda de ocho leguas de largo por cuatro de ancho (Cúneo Vidal, 1925: 199; Hemming, 1993: 272). El virrey Pedro de La Gasca (1546-1551) aceptó otorgar a Sayri Tupac todas las tierras que estaban en un triángulo formado por el valle del Apurímac, el camino real y el río Aucapampamayu. Estas tierras, en las cuales habían seiscientos indios, eran encomiendas de Hernando Pizarro, Alonso Carrasco y Gregorio Setiel (Cúneo Vidal, 1925: 200; Lohmann Villena, 1948-49: 349; Hemming, 1993: 272).

Sin embargo, el acuerdo con el virrey Pedro de La Gasca nunca ha sido cumplido. Asi, Sayri Tupac no pudo recuperar Choquequirao. No obstante, podemos preguntarnos sobre el interés de Sayri Tupac en cuanto a estas tierras en el valle del Apurímac, y especialmente a Choquequirao. Sabemos que Sayri Tupac era el jefe de la panaca del Inca Tupac Inca Yupanqui (Rostworowski, 1970: 162). ¿Eso indicaría que Choquequirao era una propiedad privada de Tupac Inca Yupanqui, al igual que Machu Picchu, que habría sido una hacienda real de Pachacuti (Rowe, 1987: 1620)? ¿El deseo de Sayri Tupac era recuperar las antiguas tierras de su bisabuelo? De momento, solo podemos plantearnos estas preguntas.

Necesitamos acordarnos de las circunstancias en que Hernando Pizarro recibió, en 1539, estas encomiendas en los valles del Apurímac, del Urubamba y del Amaybamba (Glave \& Remy, 1983: 6; Varón Gabai, 1996: 320; Julien, 2001: 249). En 1537, Manco Inca se había refugiado en la cordillera de Vilcabamba después del fracaso del cerco del Cusco en 1536. Catherine Julien (1998: 504) sugiere con razón, que la deterioración de las relaciones entre Manco Inca y los españoles habría sido la consecuencia del fracaso de las negociaciones entre los dos partidos, en cuanto a las tierras que Francisco Pizarro debía otorgar a Manco Inca como recompensa de su alianza con ellos, en el momento de su llegada al Cusco. Las tierras, llamadas «la encomienda del Inca» (Julien, 1998: 490, 501), estaban directamente vinculadas con las que poseía la dinastía Inca (Rostworowski, 1962; 1963; 1970; Villanueva Urteaga, 1970a; 1970b; Rowe, 1987). Estas tierras no habían sido otorgadas en el primer repartimiento de 1535 (Julien, 1998: 490; 2002: 184) y corresponderían, muy posiblemente, a las tierras que recibió Hernando Pizarro en los valles del Urubamba, del Amaybamba y del Apurímac.

Sea como fuere, se nota la voluntad de parte de la familia Pizarro de controlar estos tres valles, debido a su muy importante interés agrícola. En efecto, estos valles constituyen las tierras más ricas de la región del Cusco. Además, dichos valles tienen un carácter eminentemente estratégico. De hecho, son aquéllos valles que permiten entrar en la cordillera de Vilcabamba, donde estaba refugiado Manco Inca. Manifestamente, una de las preocupaciones de los Pizarro era también el control de las tierras fronterizas con las de Manco Inca. Cabe señalar también que Hernando Pizarro recibió en encomienda «el pueblo de Biticos [Vitcos] con todos sus yndios y el valle de 
Bilcabanva [Vilcabamba] con todos sus yndios» (Julien, 2001: 249; Varón Gabai, 1996: 320). Así, Hernando Pizarro era el encomendero de tierras fuera de todo control español, porque ellas estaban ocupadas directamente por los incas de Vilcabamba.

Las tierras otorgadas a Hernando Pizarro en 1539 en los valles del Apurímac, del Urubamba, del Amaybamba y en la cordillera de Vilcabamba constituyen en sí un conjunto homogéneo: son los valles que permiten penetrar en la cordillera de Vilcabamba y la cordillera misma. ¿Todo este conjunto formaba parte de la «encomienda del Inca»? Las investigaciones y las conclusiones de Julien (2001: 241) parecen indicarlo. Además, otros documentos sugieren que los asientos de Vitcos y Vilcabamba eran posesiones de Pachacuti (Rowe, 1997: 278-279). A la luz de estos datos, se puede plantear la hipótesis de que la cordillera de Vilcabamba era un territorio constituido por propiedades privadas de la dinastía Inca, que queda por investigar.

\section{CHOQUEQUIRAO DESPUÉS DE SU ABANDONO: IMPLICACIONES ARQUEOLÓGICAS}

A consecuencia del abandono de Choquequirao, la espesa ceja de montaña ocultó el conjunto arqueológico y ninguna crónica de los siglos XVI y XVII habló del sitio. En el siglo XVIII, Huertas Vallejos (1972, 203), Cosme Bueno (1957 [1768]) y Pablo José Oricain (1906 [1790]) mencionan de nuevo a Choquequirao, que tenía fama de llamarse la Ciudad de la Platería, debido a su proximidad con minas de plata ubicadas al pie del nevado que domina el sitio (ADC, Intendencia, Real Hacienda, L. 176, año 1786¹). Pero, la población de Cachora nunca olvidó Choquequirao y nació la leyenda de que el sitio, ubicado en la cordillera de Vilcabamba, símbolo de la resistencia Inca frente a los españoles, había sido el último refugio de los incas. Son estas leyendas que Eugènes de Sartiges (1851: 1040-1041), Léonce Angrand (Angrand, 1972: 33-34; Desjardins, 1858: 145), Charles Wiener (1993 [1880]: 311), Antonio Raimondi (1876: 161) y numerosos peruanos escucharon en el siglo XIX (González de la Rosa, 1908: 262; Romero, 1909: 99-101). Son estos mismos relatos que recogió también Hiram Bingham (1910: 506), más de 250 años después del abandono del sitio.

Las implicaciones arqueológicas de estos nuevos elementos etnohistóricos y las preguntas son multiples. En cuanto a la arquitectura de Choquequirao, Samanez y Zapata han observado tres fases en la evolución del complejo arqueológico: dos étapas de contrucción y una tercera con modificaciones (elementos adosados, vanos y escaleras tapados). Los autores sugieren que estas fases corresponden a la época Inca (Samanez y Zapata, 1995: 103, 108-109). Sin embargo, ¿no podrían existir, en este sitio, algunos edificios construidos en la época colonial? En los datos recogidos por Julien (2001: 250), el cacique Ataopoma de «Chuquicarando [...] con todos los yndios e prinçipales a el subjetos» tenían sus casas en el sitio. Es de preguntarse si ellos vivían en el núcleo del sitio mismo o en otros sectores, afuera del casco urbano. Futuras investigaciones arqueológicas, desarrolladas en Choquequirao, deberían proporcionar elementos a fin de identificar los sectores del sitio ocupados en la época colonial.

Para los arqueólogos, la cerámica es uno de los indicadores que permite determinar la antigüedad de un sitio arqueológico. La alfarería encontrada en Choquequirao por Copesco, el organismo peruano encargado de la puesta en valor del sitio desde 1993 (Lecoq \& Duffait, 2004: 53), no ha sido estudiada todavía y queda por investigar. Las últimas investigaciones realizadas en un sector de Choquequirao (el 9) permitieron ubicar cerámica de varias épocas (Inca, Killke y Formativa), pero no se han encontrado huellas de cerámica de estilo colonial de momento (Lecoq, 2004). Sin embargo, y como lo subraya Ann Kendall (1976: 63), sabemos que, en muchos sitios, los estilos cerámicos utilizados en la época Inca no cambiaron inmediatamente después de la

1 Agradecemos a Ronald Camala Valenzuela que nos proporcionó las referencias del manuscrito. 
conquista española, lo que obviamente dificulta la identificación de la alfarería usada al inicio del período colonial en Choquequirao. No hay que descartar tampoco la posibilidad, como lo sugiere Luis Lumbreras, que una parte de la cerámica Inca encontrada en Choquequirao, con una fuerte impronta local, podría ser el reflejo de una ocupación colonial (Lumbreras \& Wust, 2001: 31-32). En la zona periférica de Choquequirao y al nivel microrregional (cuencas de Cachora y Huanipaca), se necesita investigar los patrones de asentamiento de los sitios del Horizonte Tardío y del principio de la época colonial, y las relaciones entre éstos.

La presencia de caminos plantea problemas muy parecidos a los de la arquitectura y de la alfarería. Hace poco, el proyecto Qhapaq Ñan del Instituto Nacional de Cultura del Cusco, registró varios tramos del camino prehispánico Choquequirao-Vitcos, ubicados a 5 kilómetros al noroeste de Choquequirao y asociados a un sitio llamado Cotacoca (fig.1) (Valencia Garcia, 2002: 17-18). Por otro lado, el camino empedrado Cusco-Choquequirao, asociado con recintos para camélidos, sale del camino real a la altura de Zurite y corre al pie de los nevados; sin embargo, las investigaciones realizadas no pudieron identificar caminos empedrados entre Choquequirao y el margen izquierdo del valle del Apurímac (Duffait, 2005). Estos datos nos conducen a sugerir que las vías que conectaban Choquequirao con la ribera izquierda eran más bien unos simples senderos. Las antiguas vías que conectaban los dos margenes eran, tal vez, el camino actual Cachora-Choquequirao y el sendero que cruza el Apurímac al pie de Choquequirao. Es de preguntarse, entonces, si todas estas vías de acceso, fueron reutilizadas en la época colonial.

\section{CONCLUSIONES}

En 1539, dos años después del retiro de Manco Inca en la cordillera de Vilcabamba, la familia Pizarro se otorgó las mejores tierras de la región del Cusco que todavía no habían sido distribuidas en el primer repartimiento del año 1535. Hernando Pizarro recibió varias tierras y pueblos en los valles del Amaybamba, del Urubamba y del Apurímac. En el valle del Urubamba, los pueblos de Tambo (Ollantaytambo) y Picho (Machu Picchu) fueron encomendados a Hernando Pizarro. Por otro lado, recibió en el valle del Apurimac los pueblos de Urco, Sayhuite y Choquequirao. Estos datos publicados por Rafael Varón Gabai y Catherine Julien, y confrontados con otras fuentes, nos indican que los españoles tenían conocimiento de Choquequirao que estaba en «tierra de paz» y afuera del control de los incas de Vilcabamba.

El análisis de los documentos de archivos parece indicar que Choquequirao fue reducido en el pueblo de Cachora al fin del siglo XVI, conjuntamente con los pueblos de Sayhuite y Urco. El pueblo de reducción de Cachora fue creado, probablemente, en la época del virrey García Hurtado de Mendoza (1590-1596). Sin embargo, eso no significa que Choquequirao haya sido totalmente abandonado durante este virreinato. De hecho, si Cachora no fue una reducción exitosa inmediatamente, lo que ocurrió a menudo, podemos suponer que la gente que vivía en Choquequirao habría podido quedarse en el sitio durante parte del siglo XVII. De momento, los elementos disponibles nos permiten plantear únicamente estas posibilidades, sin poder proponer una fecha exacta en cuanto al abandono de Choquequirao.

No obstante, podemos notar que durante más de 80 años por lo menos, entre 1539 y 1618 , los cargos de caciques recayeron sobre las mismas familias: Ache de Sayhuite, Ataopoma de Choquequirao y Ataouuco de Urco. Estos datos parecen indicar que los españoles utilizaron las estructuras de poder anteriores a la conquista, a fin de controlar y sujetar la población indígena. Sin embargo, son menos conocidos y quedan por investigar los posibles vínculos entre Choquequirao y la dinastía Inca, y especialmente con la panaca de Tupac Inca Yupanqui.

A la luz de estos nuevos datos se abren nuevas perspectivas y se hacen nuevas preguntas relativas a la ocupación colonial de Choquequirao, hasta hoy desconocida, y verificable mediante un análisis minucioso del material arqueológico, y a través del estudio arquitectónico riguroso de los diferentes sectores que componen el sitio. Confiamos en los nuevos proyectos arqueológicos, desarrollados en Choquequirao por Perú y Francia, a fin de contestar estas preguntas. 


\section{Referencias citadas}

\section{Fuentes manuscritas}

Archivos Departamentales del Cusco (ADC):

- Cabildo, Legajo 3, Cuaderno 25, año 1637.

- Colegio de Ciencias, Legajo 11, Cuaderno 15, años 1654-1825.

- Intendencia, Real Hacienda, Legajo 176, año 1786.

Archivo General de la Nación, Lima (AGN):

- Derecho Indigena, Legajo 25, Cuaderno 451, año 1791

Biblioteca Nacional del Perú, Lima (BNP):

- B 753, año 1618.

AMADO GONZÁLES, D., 1999 - Introducción al estudio histórico de los títulos de la Hacienda de Punchaopuquio, Curahuasi-Cusco. Revista del Archivo Departamental del Cusco, 14: 25-43.

ANGRAND, L., 1972 - Imagen del Perú en el siglo XIX, 285 p.; Lima: Editor Carlos Milla Batres.

BINGHAM, H., 1910 - The ruins of Choqquequirau. American Anthropologist, 12: 505-525.

BUENO, C., 1951 [1768] - Geografía del Perú virreinal, siglo XVIII, 140 p.; Lima: Daniel Valcarcel editor.

CÚNEO VIDAL, R., 1925 - Historia de las guerras de los últimos Incas peruanos contra el poder español (1535-1572), 313 p.; Barcelona: Casa Editorial Maucci.

DESJARDINS, E., 1858 - Le Pérou avant la conquête espagnole, 186 p.; Paris: A. Bertrand.

DUFFAIT, E., 2005 - La red vial prehispánica de la cordillera de Vilcabamba (Departamento del Cusco, Perú): un esbozo de estudio preliminar. In: De l'Altiplano mexicain à la Patagonie: Travaux et recherches à l'Université de Paris 1 (Giorgi, C., ed.): 79-88; Oxford: Archaeopress. British Archaeological Reports, International Series 1389.

FORNEE, N., 1965 [1586] - Descripción de la tierra del corregimiento de Abancay, de que es corregidor Niculoso de Fornee. In: Relaciones Geográficas de Indias (Jiménez de la Espada, M., ed.): 16-30; Madrid: Atlas. Biblioteca de Autores Españoles, 184.

GLAVE, L. M. \& REMY, M. I., 1983 - Estructura agraria y vida rural en una región andina: Ollantaytambo entre los siglos XVI y XIX, 584 p.; Cusco: Centro de Estudios Rurales Andinos Bartolomé de Las Casas.

GONZÁLEZ DE LA ROSA, M., 1908 - A propos de la redécouverte de la ville antique de Choquéquirao sur la rive droite de l'Apurimac (Pérou). Journal de la Société des Américanistes, 5: 261-264.

HEMMING, J., 1993 - The conquest of the Incas, 624 p.; London: Papermac Pan Macmillan.

HUERTAS VALLEJOS, L., 1972 - Memorial acerca de las cuatro ciudades inkas situadas entre los ríos Urubamba y Apurímac. Historia y Cultura, 6: 203-205.

INSTITUTO GEOGRÁFICO MILITAR, 1946 - Hoja de Curahuasi, 13 g, 1/200 000; Lima: Instituto Geográfico Militar.

JULIEN, C., 1998 - La encomienda del Inca. In: Actas del IV Congreso Internacional de Etnohistoria, 2: 489-516; Lima: Pontificia Universidad Católica del Perú, Fondo Editorial.

JULIEN, C., 2001 - Inca estates and the encomienda: Hernando Pizarro's holdings in Cusco. Andean Past, 6: 229-275. 
JULIEN, C., 2002 - Diego Maldonado y los Chancas. Revista Andina, 34: 183-197.

KENDALL, A., 1976 - Descripción e inventario de las formas arquitectónicas inca. Patrones de distribución e inferencias cronológicas. Revista del Museo Nacional, 42: 13-96.

KENDALL, A., 1980 - Proyecto Cusichaca. Etnohistoria, reconocimiento (Tomo 3), 129 p.; Cusco: Instituto Nacional de Cultura.

LECOQ, P., 2004 - Choqek'iraw, un site formatif ? Résultats préliminaires de la campagne de fouilles menées sur ce site en août 2004. Bulletin de I'Institut Français d'Etudes Andines, 33 (2): 379-383.

LECOQ, P. \& DUFFAIT, E., 2004 - Choqek'iraw, un nouveau Machu Picchu ? Archéologia, 411: 50-63.

LOHMANN VILLENA, G., 1948-1949 - El señorío de los Marqueses de Santiago de Oropesa en el Perú. Anuario de Historia del Derecho Español, 19: 347-458.

LUMBRERAS, L. \& WUST, W., 2001 - Choqequirau: santuario histórico y ecológico, 51 p.; Lima: Fundación Telefónica.

MATIENZO, J., 1967 - Gobierno del Perú (1567), 366 p.; Lima: Institut Français d'Études Andines. Travaux de I'Institut Français d'Études Andines, 11.

MIRANDA, C., 1906 [1583] - Relación de los oficios que se proveen en el reino del Perú. In: Juicio de limites entre el Perú y Bolivia (Maurtua, V., ed.), 1: 151-180; Barcelona: Imprenta de Heinrich y Comp.

MIRANDA, C., 1975 [1583] - Tasa de la visita general de Francisco de Toledo. In: Tasa de la visita general de Francisco de Toledo (Cook, N.D. ed.): 3-295; Lima: Universidad Nacional Mayor de San Marcos.

MIRANDA VALENZUELA, J., 2002 - Abancay: provincia andina, una mirada del pasado al presente, 592 p.; Abancay: Apurímac.

MURÚA, M., 1962 [1611] - Historia general del Perú, 279 p.; Madrid: Instituto Gonzalo Fernández de Oviedo. Colección Joyas bibliográficas: Biblioteca Americana Vetus, 1.

ORICAIN, P. J., 1906 [1790] - Compendio breve de discursos varios sobre diferentes materias y noticias geográficas comprehensivas a este obispado del Cuzco. In: Juicio de limites entre el Perú y Bolivia (Maurtua, V., ed.), 11: 319-377; Barcelona: Imprenta de Heinrich y Comp.

PUENTE BRUNKE, J., 1992 - Encomienda y encomenderos en el Perú: estudio social y político de una institución colonial, 536 p.; Sevilla: Excma. Diputación Provincial de Sevilla.

RAIMONDI, A., 1876 - El Perú (Tomo 2), 475 p.; Lima: Imprenta del Estado - Gil.

ROMERO, C., 1909 - Informe del señor Carlos A. Romero, individuo de número del Instituto, sobre las ruinas de Choqquequirau. Revista Histórica, 4: 87-103.

ROSTWOROWSKI, M., 1962 - Nuevos datos sobre tenencia de tierras reales en el incario. Revista del Museo Nacional, 31: 130-159.

ROSTWOROWSKI, M., 1963 - Dos manuscritos inéditos con datos sobre Manco II, tierras personales de los Incas y mitimaes. Nueva Corónica, 1: 223-239.

ROSTWOROWSKI, M., 1970 - El repartimiento de doña Beatriz Coya en el valle de Yucay. Historia y Cultura, 4: 153-267.

ROWE, J., 1987 - Machu Picchu a la luz de documentos del siglo XVI. Kuntur: Perú en la cultura, 4: 12-20.

ROWE, J., 1997 - Las tierras reales de los Incas. In: Arqueología, antropología e historia en los Andes. Homenaje a María Rostworowski (Varón Gabai, R. \& Flores Espinoza, J., eds.): 277-287; Lima: Instituto de Estudios Peruanos-Banco Central de Reserva del Perú.

SAMANEZ, R. \& ZAPATA, J., 1995 - El conjunto arqueológico inka de Choquequirao. Andes, 1: 97-114.

SARTIGES, E., 1851 - Voyage dans les républiques de l'Amérique du Sud. Ecrit sous le pseudonyme de E. De Lavandais. Revue des Deux Mondes, 21 (10): 1019-1059. 
TITU CUSI YUPANQUI, I., 1916 [1570] - Relación de la conquista del Perú y hechos del Inca Manco II, 154 p.; Lima: Sanmarti. Notas bibliográficas y concordancias del texto por Horacio H. Urteaga, biografía de Tito Cusi Yupanqui por Carlos A. Romero. Colección de libros y documentos referentes a la historia del Perú, 2.

TOLEDO, F., 1989 - Francisco de Toledo: disposiciones gubernativas para el virreinato del Perú. 1575-1580, 507 p.; Sevilla: Escuela de estudios hispano-americanos - Monte de Piedad y Caja de ahorros de Sevilla. Introducción: Lohmann Villena, Guillermo; Transcripción: Sarabia Viejo, María Justina.

VACA DE CASTRO, C., 1908 [1543] - Ordenanzas de tambos, distancia de unos a otros, modo de cargar a los indios y obligaciones de las justicias respectivas. Hecho en el Cuzco el 31 de mayo 1543. Revista Histórica, 3: 427-492.

VALENCIA GARCIA, Z., 2002 - Informe técnico trimestrial del proyecto de identificación, catastro y evaluación del sistema vial prehispánico Santa Teresa-Choquekirao-Vitcos, 70 p.; Cusco: Instituto Nacional de Cultura. Dirección departamental Cusco. Proyecto Qhapaq Ñan.

VARÓN GABAI, R., 1996 - La ilusión del poder: apogeo y decadencia de los Pizarro en la conquista del Perú, 450 p.; Lima: Instituto de Estudios Peruanos-Institut Français d'Etudes Andines.

VARÓN GABAI, R., 1998 - Las encomiendas de Hernando Pizarro. In: Actas del IV Congreso Internacional de Etnohistoria, 2: 535-549; Lima: Pontificia Universidad Católica del Perú, Fondo Editorial.

VILLANUEVA URTEAGA, H., 1970a - Documentos sobre Yucay en el siglo XVI. Revista del Archivo Histórico del Cuzco, 13: 1-148.

VILLANUEVA URTEAGA, H., 1970b - Información ad perpetuam dada en 13 de enero de1567 ante la real justicia de la ciudad del Cuzco, reino del Perú a pedimento de la muy ilustre señora doña María Manrrique Coya, vecina de dicha ciudad. Revista del Archivo Histórico del Cuzco, 13: 149-184.

WIENER, C., 1993 [1880] - Perú y Bolivia: relato de viaje, 859 p.; Lima: Institut Français d'Etudes Andines-Universidad Nacional Mayor de San Marcos. Travaux de I'Institut Français d'Etudes Andines, 56. 